\title{
Absolute timing of the Crab Pulsar at optical wavelengths with superconducting tunneling junctions
}

\author{
T. Oosterbroek ${ }^{1}$, J. H. J. de Bruijne ${ }^{2}$, D. Martin ${ }^{1}$, P. Verhoeve ${ }^{1}$, M. A. C. Perryman ${ }^{2}$, C. Erd ${ }^{1}$, and R. Schulz ${ }^{2}$ \\ ${ }^{1}$ Science Payload and Advanced Concepts Office, ESA, ESTEC, Postbus 299, 2200 AG Noordwijk, The Netherlands \\ e-mail: toosterb@rssd.esa.int \\ 2 Research and Scientific Support Department of ESA, ESTEC, Postbus 299, 2200 AG Noordwijk, The Netherlands
}

Received 23 March 2006 / Accepted 24 May 2006

ABSTRACT

\begin{abstract}
Aims. We have observed the Crab Pulsar in the optical with S-Cam, an instrument based on Superconducting Tunneling Junctions (STJs) with $\mu$ s time resolution. Our aim was to study the delay between the radio and optical pulse.

Methods. The Crab Pulsar was observed three times over a time span of almost 7 years, on two different locations, using three different versions of the instrument, and using two different GPS units.

Results. We consistently find that the optical peak leads the radio peak by $49 \pm 90,254 \pm 170$, and $291 \pm 100 \mu$ s. On assumption of a constant optical lead, the weighted-average value is $\sim 170 \mu \mathrm{s}$, or when rejecting (based on a perhaps questionable radio ephemeris) the first measurement, $273 \pm 100 \mu \mathrm{s}$.
\end{abstract}

Key words. stars: pulsars: individual: Crab Pulsar (PSR J0534+2200)

\section{Introduction}

Precise timing of pulsar light curves throughout the electromagnetic spectrum is a powerful tool to constrain theories of the spatial distribution of various emission regions. In recent years, it has become clear that the main and secondary pulses of the Crab Pulsar (PSR J0534+2200) are not aligned in time at different wavelengths. X-rays are leading the radio pulse by reported values of $344 \pm 40 \mu$ s (Rots et al. 2004; RXTE data) and $280 \pm 40 \mu \mathrm{s}$ (Kuiper et al. 2003; INTEGRAL data) and $\gamma$-rays are leading the radio pulse by $241 \pm 29 \mu$ s (Kuiper et al. 2003; EGRET data. The uncertainty in this value does not include the EGRET absolute timing uncertainty of better than $100 \mu \mathrm{s}$ ). At optical wavelengths, the observations present a less coherent picture. Sanwal (1999) has reported a time shift of $140 \mu$ s (optical leading the radio). The uncertainty in this value is $20 \mu$ s in the determination of the optical peak and $75 \mu \mathrm{s}$ in the radio ephemeris. Shearer et al. (2003) have reported a lead of $100 \pm 20 \mu$ s for simultaneous optical and radio observations of giant radio pulses. Golden et al. (2000) have reported that the optical pulse trails the radio pulse by $\sim 80 \pm 60 \mu$ s. Romani et al. (2001) conclude that the radio and optical peaks are coincident to better than $30 \mu \mathrm{s}$, but their error excludes the uncertainty of the radio ephemeris $(150 \mu \mathrm{s})$. The internal inconsistency of these results - if we assume that the optical-radio delay is constant - has prompted us to look into this matter in detail using recent observations in combination with earlier data.

\section{Observations}

Observations were obtained with S-Cam3 in November 2005 on the ESA Optical Ground Station (OGS) telescope on Tenerife and with S-Cam2 in October 2000 and S-Cam1 in February 1999 (see Perryman et al. 1999) on the WHT telescope on La Palma (Table 1). All S-Cam instruments are based on Superconducting
Tunneling Junctions (STJs), which register individual photons with high time resolution and moderate spectral resolution (see e.g. Martin et al. 2003). Times are provided by a GPS unit which yields an accuracy better than $1 \mu \mathrm{s}$ with respect to UTC in individual photon arrival times. The time resolution of the instrument was $5 \mu$ s for S-Cam1 and S-Cam 2 and $1 \mu$ s for S-Cam3.

\section{Analysis and results}

The time tags of all photons were converted from UTC to Barycentric Dynamical Time (TDB) at the solar system barycenter (a process we refer to as "barycentering"). This process involves conversion from UTC to TAI (by - for our observations adding 32 leap seconds) and subsequent conversion from TAI to TT by adding the constant offset of $32.184 \mathrm{~s}$. TT is then converted TDB. (Although the IAU officially replaced TDB by TCB in 1991, they reckoned that, where continuity with previous work is desirable, TDB may be still be used.) Furthermore, a correction from the observatory to the Earth centre and a correction for the gravitational propagation delay are applied. We have used the JPL DE200 ephemeris (and not the more recent DE405 ephemeris) to perform the barycentering, since we compare our data against observations of the Crab Pulsar in the radio, which are also barycentered using this ephemeris. WGS84 coordinates of the telescopes were obtained with a GPS unit. These geodetic coordinates were converted into geocentric coordinates to obtain the proper observatory to Earth centre correction.

We have folded our data using the $P, \dot{P}$, and arrival times (at infinite frequency) from the Crab radio ephemeris (Lyne et al. 2005). The folded profiles are displayed in Fig. 1. An important parameter from this ephemeris is the uncertainy in radio timing, which we denote with $\sigma_{\text {radio }}$. This uncertainty is composed of different elements which can not all be treated as a statistical error that reduces when averaged over multiple observations. The 
Table 1. Log of observations.

\begin{tabular}{ccccc}
\hline \hline Instrument & Observation period (YYYY-MM-DD) & Exposure (s) & Observatory & GPS Instrument \\
\hline S-Cam1 & 1999-02-02-1999-02-04 & 4800 & WHT La Palma (4.2 m) & HP 58503A \\
S-Cam2 & $2000-10-01-2000-10-02$ & 3317 & WHT La Palma (4.2 m) & HP 58503A \\
S-Cam3 & $2005-11-12$ & 2616 & OGS Tenerife (1 m) & Zyfer GPStarplus Model 565 \\
\hline
\end{tabular}
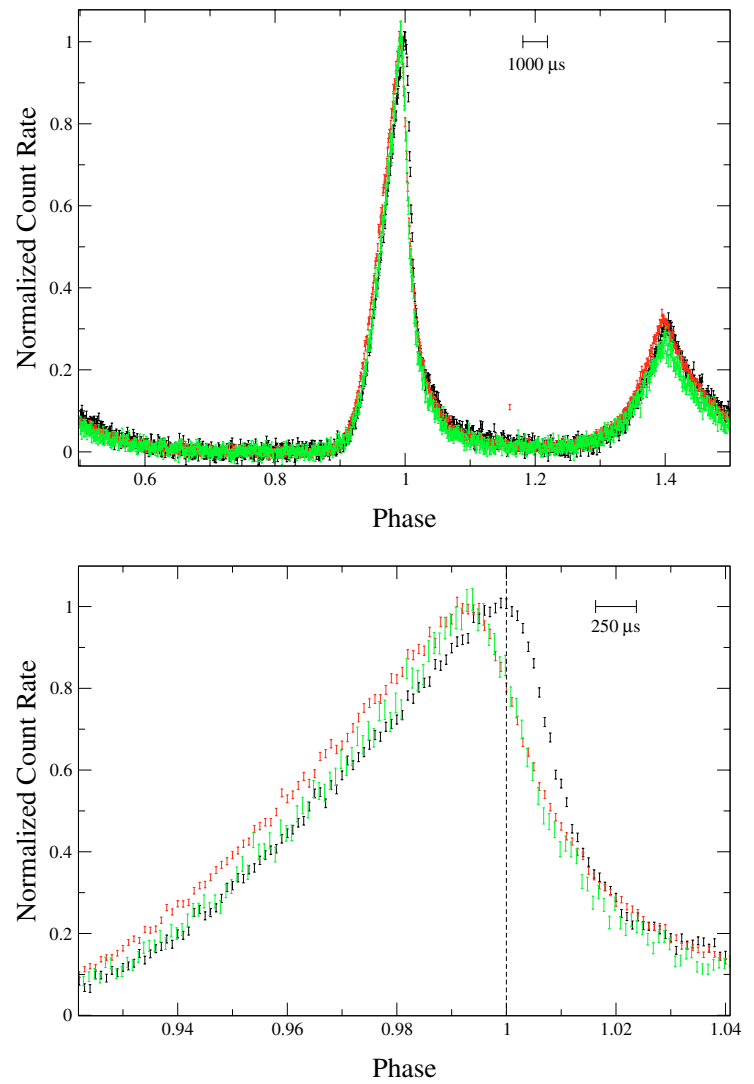

Fig. 1. The three renormalized profiles: S-Cam1 in black, S-Cam2 in red, S-Cam3 in green (in the electronic version). In the top panel, the total profile is displayed. The bottom panel shows a zoom-in on the main peak. The statistical noise is largest for the S-Cam3 observation, due to the smaller telescope and shorter exposure time. Phase 0 (or 1) is defined as the peak of the radio ephemeris; this phase is indicated by the vertical dashed line.

most important components are unmodeled delays and imperfect polarization calibration $(\sim 40 \mu \mathrm{s})$, uncertainties in the delay due to scattering $(\sim 20 \mu \mathrm{s})$, and variations in the daily time-of-arrival measurements with rms residuals of the order 20-50 $\mu$ s.

No default method exists for determining the position of the peak of the profile. We follow the method of Rots et al. (2004), which differs, however, from the choice of other authors (e.g. Kuiper et al. 2003). The folded pulse profile (subdivided into 1000 phase bins) was fitted in a phase range of 0.01 centered around the peak using an iterative approach, allowing the phase range in use to evolve with the fitted peak position. The model used to determine the peak was a Lorentzian with normalization, width, and center as free parameters. This choice is somewhat arbitrary and different fitting functions (e.g. a Gaussian or a parabola) return slightly different results. Variations, however, are always much less than the uncertainty in the radio ephemeris, the final number to which we compare against. We have also studied the behaviour of the peak position obtained with different phase ranges. These experiments show a consistent behaviour: due to the asymmetric shape of the pulse profile, the phase of the peak shifts to a higher value as the phase range is decreased but flattens off for phase ranges smaller than 0.01 . We estimate that the uncertainty introduced by using a finite phase range of 0.01 (instead of an ideal, infinitely small phase range) is $10 \mu \mathrm{s}$. Our results are summarised in Table 2. In all cases, the optical pulse leads the radio pulse. The time shift found from S-Cam1 data is clearly the smallest, while the value from $\mathrm{S}-\mathrm{Cam} 2$ data is affected by a substantially larger $\sigma_{\text {radio }}$.

We have also determined the position of the secondary peak using the same algorithm as above but with twice the phase range (i.e. a width of 0.02). This increased range is required since the secondary peak is less sharp (resulting in almost no curvature in a 0.01 phase range) and has a lower signal to noise ratio. The results (see Table 2) are consistent with a constant phase difference between the primary and secondary peak. This confirms that the $\mathrm{S}-\mathrm{Cam} 1$ pulse profile is shifted (compared to the S-Cam 2 and S-Cam3 data points): not only the main peak arrives later, but also the secondary, indicating a shift of the whole pulse profile, not a change in the main peak only.

In order to compare the results of the three S-Cam campaigns, the three different pulse profiles were scaled to match each other. The level in the phase range $0.7-0.8$ was calculated as well as the peak level determined from the mean of the three highest bins. The profile was renormalized such that the peak is at 1 and the base level at 0 . This scaling assumes that there are no significant counts from the pulsar in the $0.7-0.8$ phase range and that all other photons (sky background, nebula background, and noise photons) are distributed equally in phase.

The normalised profiles look similar, albeit the S-Cam1 profile looks a bit different at the peak: the leading part of the peak is almost identical to the S-Cam3 profile (up to phase 0.98), but then starts to move away and peaks later than the S-Cam2 and S-Cam3 profiles. We have investigated the possibility that the $P$ and $\dot{P}$ which have been used to fold the data are sub-optimum by performing a period search on our data. However, the solution hence found is fully consistent with the radio ephemeris, with the latter having a higher precision. Although we do not have evidence for a large colour-dependence of the profile shape, small differences in the detailed shape of the pulse profile may be explained by the different spectral response and array size of the different generations of the S-Cam instrument, possibly in combination with different sky-background levels and atmospheric conditions under which the observations were acquired.

We have also investigated, by exploiting the intrinsic spectral resolution of STJs, whether there is a phase difference in the peak between the "red" and the "blue". We have selected the $\sim 30 \%$ photons with highest energy $(\sim 400-500 \mathrm{~nm})$ and the $\sim 30 \%$ photons with lowest energy $(\sim 600-760 \mathrm{~nm})$. Because of the limited spectral resolution of the S-Cam instrument, this approach yields a cleaner colour separation than splitting the photons in two adjacent bands, each containing $50 \%$ of all events. We then determine the delay between the photons in the red band with respect to the photons in the blue band by doing a crosscorrelation of the whole main peak profile. We find that the red 
Table 2. Radio to optical delays. The used parameters from the Crab monthly ephemeris are also displayed (converted into $P$ and $\dot{P}$ ). The number of digits displayed is higher than the uncertainty. The $\sigma_{\text {radio }}$ quoted is the uncertainty in the radio ephemeris as published in the Crab monthly ephemeris. The separation is defined as the difference (in phase) between the primary and secondary peak.

\begin{tabular}{ccccccc}
\hline \hline Instrument & Delay $(\mu \mathrm{s})$ & $\sigma_{\text {radio }}(\mu \mathrm{s})$ & Separation & $P(\mathrm{~s})$ & $\dot{P}\left(10^{-13} \mathrm{~s} \mathrm{~s}^{-1}\right)$ & Epoch $(\mathrm{MJD})$ \\
\hline S-Cam1 & $49 \pm 10$ & 80 & $0.4056 \pm 0.0009$ & 0.03349352792448 & 4.20554737 & 51224.000000352002 \\
S-Cam2 & $254 \pm 8$ & 160 & $0.4052 \pm 0.0005$ & 0.03351561813489 & 4.20539152 & 51832.000000238553 \\
S-Cam3 & $291 \pm 13$ & 90 & $0.4065 \pm 0.0017$ & 0.03358309316492 & 4.20593301 & 53689.000000194479 \\
\hline
\end{tabular}

photons lag by $-1 \pm 2 \mu$ s for S-Cam1, $4 \pm 4 \mu$ s for S-Cam2, and $5 \pm 10 \mu$ s for $\mathrm{S}-\mathrm{Cam} 3$. This does not represent a significant time shift and can be compared to the results of Sanwal (1999) who found a delay between the $U$ and $R$ band of $10 \pm 4 \mu$ s and Golden et al. (2000) who found that the peaks in $B$ and $V$ are coincident within $10 \mu \mathrm{s}$.

\section{Checks on correctness of times}

The following checks were made:

GPS time: it was verified that both GPS units provide the same time to within $0.1 \mu \mathrm{s}$, the typical intrinsic GPS time jitter.

GPS position: the correct reading of the S-Cam3 GPS was verified by the GPS present at the OGS telescope. This GPS presents the position in Earth-Centered, Earth-Fixed (ECEF) coordinates $(X, Y, Z)=(5390284,-1597899,3007075) \mathrm{m}$. The reading of the S-Cam 3 GPS (latitude $28^{\circ} 18^{\mathrm{m}}$.0640, longitude $16^{\circ} 30^{\mathrm{m}} .7133$ and altitude $2447.02 \mathrm{~m}$ ) was converted into ECEF coordinates which resulted in $(X, Y, Z)=(5390281$, $-1597891,3007083) \mathrm{m}$. These coordinates differ by $12 \mathrm{~m}$, consistent with the separation between the two GPS antennas.

Period-folding software: two different approaches of folding the data were followed and it was verified that they gave identical results. The first approach is to fold the data with the period, period derivative, and epoch as given by the Lyne et al. (2005) radio-timing results. The second approach is to determine, by phase folding, the "current period" (i.e. at the time of the observation) and fold the data on that period without taking the period derivative into account, which is not necessary for such short datasets. The phase zero (or epoch) close to the time of the observation should be independently calculated using the radio ephemeris. While in theory both methods should give identical results, this is by no means guaranteed in practice: since the time of observation can be 15 days away from the ephemeris epoch, the calculation of the phase using the period and period derivative requires high precision. It was verified that this requirement on precision is met by the FTOOLS "efold" program.

Barycentering software: using the detailed examples provided in Lyne et al. (2005), it was verified that our barycentering code works correctly at the $5 \mu$ s level (dominated by corrections in TT to TDB: relativistic corrections from the geoid to the solar system barycenter).

Crab coordinates: for the barycentering of the Crab, the coordinates used by Lyne et al. are: $05^{\mathrm{h}} 34^{\mathrm{m}} 31^{\mathrm{s}} .97232$, $+22^{\circ} 00^{\prime} 52^{\prime \prime}$.0690 (J2000). For each monthly ephemeris, this fixed position is used as an assumed position for the pulsar at the relevant epoch. The known proper motion of $\sim 18 \mathrm{mas} / \mathrm{yr}$ is ignored, and the effect of this proper motion on the true position is incorporated into the solution of the period, period derivative and arrival time (C.A. Jordan, private communication). Our barycentric correction assumes the same reference position as the relevant monthly ephemeris, so that consistent arrival times are obtained. The erroneous use of proper-motion corrected coordinates in the barycentering would have a significant effect: inclusion of a proper motion of $18 \mathrm{mas} / \mathrm{yr}$ would imply a time difference of $\sim 17 \mu$ s per year, or $\sim 100 \mu$ s for the 2005 observation.

Instrumental effects: the observation of delays/shifts at the level of a few hundred $\mu$ s and the realization that this time scale is comparable to the average time between the events for the mean count rate during the Crab observations, raises the possibility that photons could have the time tag belonging to the previous photon. This wrong association could either be caused by software or by instrumental effects. Both possibilities were studied in detail and rejected: we are confident that our photons have correct time tags.

Instrumental effects (S-Cam2): the S-Cam2 data suffered from a hardware problem which resulted in corruption of time tags of $75 \%$ of all events, randomly distributed in time. Since this problem is fully deterministic and understood, photons with corrupted time tags have been filtered out. The remaining $25 \%$ of photons, which have reliable time tags, have been used for this analysis.

Instrumental delays (S-Cam1 and S-Cam2): in S-Cam1 and S-Cam2, incoming photons generate electronic signals from which the photon energy and arrival time can be extracted. The arrival time of a photon is defined as the moment in time that the electronic signal rises above a certain threshold. The delay of the registered arrival time compared to the true arrival time is less than $10 \mu \mathrm{s}$, and corrections for this delay have not been made for S-Cam1 and S-Cam2 data.

Instrumental delays (S-Cam3): in S-Cam3, each photon gives rise to a bi-polar signal in the detector electronics chain. The zero-crossing of this signal is used to time tag the photon. The delay of this moment compared to the true time the photon entered the detector has been calibrated to be $66.0 \pm 1.0 \mu$ s (fully consistent with the value of $66.7 \mu$ s obtained from simulations of the instrument). All S-Cam3 time tags have been corrected for this (fixed) delay.

\section{Discussion and conclusions}

We have observed the Crab Pulsar with three different generations of the S-Cam instrument, on two locations, using two GPS units. We consistently find that the optical pulse is leading the radio pulse. However, the amount by which the optical is leading the radio differs from observation to observation. When comparing the different results as plotted in Fig. 2 which are obtained with the Jodrell Bank ephemeris, one should take into account that $\sigma_{\text {radio }}$ contains a systematical component (of $\sim 40 \mu \mathrm{s}$ ) affecting all these measurements in the same way. If we subtract the $40 \mu \mathrm{s}$, for the purpose of comparing the results, from $\sigma_{\text {radio }}$ and add the remainder, in quadrature, to our measurement uncertainties, we obtain the following values for S-Cam1, S-Cam2, and S-Cam3: $49 \pm 41,254 \pm 120$, and $291 \pm 50 \mu \mathrm{s}$.

From the last two observations (S-Cam2 and S-Cam3), we then determine an average lead of $273 \pm 65 \mu$ s. The uncertainty in this determination is dominated by uncertainties in the radio 


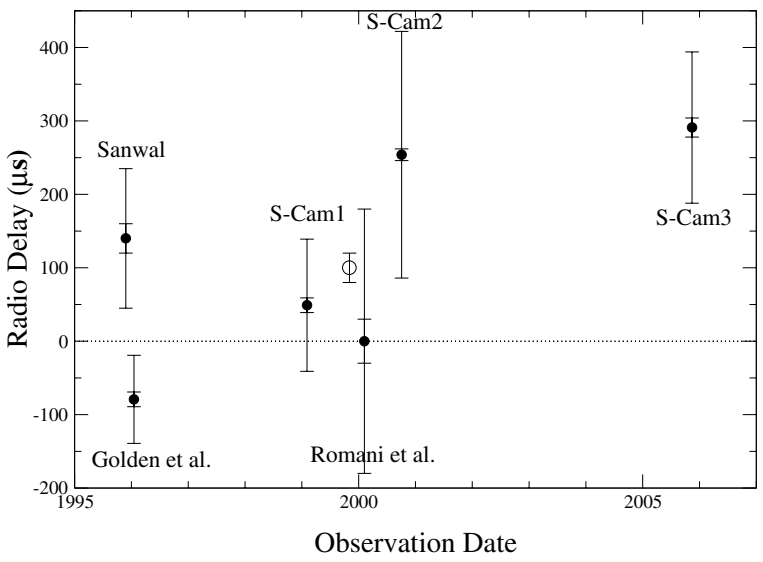

Fig. 2. Our three results combined with literature results in the optical. For all results, the uncertainties were obtained by linear addition (i.e. not quadratic) of the uncertainty in the peak determination and the uncertainty in the radio ephemeris. Uncertainties on the peak determination alone are also plotted. The open symbol refers to Shearer et al. (2003), who observed giant radio pulses.

ephemeris. The result in X-rays for the same two epochs is $370 \pm$ $40 \mu$ s (Rots, private communication).

From our data, we cannot rigorously exclude the possibility that the delay between the radio and optical peak evolves with time. The S-Cam 1 result $(49 \pm 90 \mu$ s; the uncertainty has been determined by a linear addition of the uncertainty in the peak determination and $\sigma_{\text {radio }}$ ) in particular is slightly deviant compared to the S-Cam 2 and S-Cam3 data points $(254 \pm 170$ and $291 \pm$ $100 \mu \mathrm{s})$. However, the radio observations at the times of the S-Cam1 observations have a high dispersion measure - a higher value has not been observed after February 1999. As a result, the radio-ephemeris uncertainty $(80 \mu \mathrm{s})$ might have been slightly underestimated: variations in the dispersion measure could introduce a somewhat higher uncertainty than the standard $20 \mu \mathrm{s}$ (Lyne et al. 1993). We note that Rots et al. (2004) have discarded their contemporaneous (X-ray) data point, because the timing ephemeris is somewhat suspect (the GRO version has a high second derivative indicative of a questionable fit). In the following we will therefore only consider the S-Cam 2 and S-Cam 3 data points. We emphasize here that corrections for arrival times at infinite frequency (which are substantial: $\sim 0.6 \mathrm{~s}$ at $610 \mathrm{MHz}$ ) depend on the dispersion measure. The only (literature) data point which is clearly inconsistent with all observations is that of Golden et al. (2000). We have no explanation for this.

Our value of the optical phase difference between the main and secondary pulse of $0.4054 \pm 0.0004$ is not consistent with the X-ray value from Rots et al. (2004) of $0.4001 \pm 0.0002$ periods. This implies that the details of the pulse profile are different in X-rays and at optical wavelengths.

Our time shift of $273 \pm 65 \mu$ s is somewhat smaller than, but consistent with, the time shift as obtained from X-ray measurements. A time shift of $\sim 270 \mu$ s indicates that possibly (in a simple geometrical model ignoring relativistic effects) the optical radiation is formed $\sim 90 \mathrm{~km}$ higher in the magnetosphere than the radio emission. Alternatively, the difference in phase of $\sim 0.008$ could be interpreted as an angle between the radio and optical beam of $\sim 3^{\circ}$.

Ideally, a simultaneous radio-optical observation at high frequency should be performed. With an observation like this uncertainties resulting from corrections for interstellar scattering are minimized, and the accuracy will effectively be limited by systematic effects ( $\sim 40$ s for Jodrell Bank).

Acknowledgements. We thank Dr. D. Sanwal for providing details about his results. We thank the referee, Dr. A. Rots, for useful comments.

\section{References}

Golden, A., Shearer, A., Redfern, R. M., et al. 2000, ApJ, 363, 617

Kuiper, L., Hermsen, W., Walter, R., \& Foschini, L. 2003, A\&A, 411, L31

Lyne, A. G., Pritchard, R. S., \& Graham-Smith, F. 1993, MNRAS, 265, 1003

Lyne, A. G., Jordan, C. A., \& Roberts, M. E. 2005, Crab Monthly Ephemeris available on http://www.jb.man.ac.uk/ pulsar/crab.html

Martin, D. D. E., Verhoeve, P., den Hartog, R. H., et al. 2003, Proc. SPIE, 4841, 805

Perryman, M. A. C., Favata, F., Peacock, A., Rando, N., \& Taylor, B. G. 1999, A\&A, 346, L30

Romani, R. W., Miller, A. J., Cabrera, B., et al. 2001, ApJ, 563, 221

Rots, A. H., Jahoda, K., \& Lyne, A. G. 2004, ApJ, 605, L129

Sanwal, D. 1999, Ph.D. Thesis, Univ. Texas at Austin

Shearer, A., Stappers, B., O'Connor, P., et al. 2003, Science, 301, 493 